\title{
Rate-Splitting for Multicarrier Multigroup Multicast: Precoder Design and Error Performance
}

\author{
Hongzhi Chen, De Mi, Member, IEEE, Tong Wang, Zheng Chu, Member, IEEE, Yin Xu, Dazhi He, \\ Pei Xiao, Senior Member, IEEE
}

\begin{abstract}
Employing multi-antenna rate-splitting (RS) at the transmitter and successive interference cancellation (SIC) at the receivers, has emerged as a powerful transceiver strategy for multi-antenna networks. In this paper, we design RS precoders for an overloaded multicarrier multigroup multicast downlink system, and analyse the error performance. RS splits each group message into degraded and designated parts. The degraded parts are combined and encoded into a degraded stream, while the designated parts are encoded in designated streams. All streams are precoded and superimposed in a non-orthogonal fashion before being transmitted over the same time-frequency resource. We first derive the optimized RS-based precoder, where the design philosophy is to achieve a fair user group rate for the considered scenario by solving a joint max-min fairness and sum subcarrier rate optimization problem. Comparing with other precoding schemes including the state-of-the-art multicast transmission scheme, we show that the RS precoder outperforms its counterparts in terms of the fairness rate, with Gaussian signalling, i.e., idealistic assumptions. Then we integrate the optimized RS precoder into a practical transceiver design for link-level simulations (LLS), with realistic assumptions such as finite alphabet inputs and finite code block length. The performance metric becomes the coded bit error rate (BER). In the system under study, low-density parity-check (LDPC) encoding is applied at the transmitter, and iterative soft-input soft-output detection and decoding are employed at the successive interference cancellation based receiver, which completes the LLS processing chain and helps to generate the coded error performance results which validate the effectiveness of the proposed RS precoding scheme compared with benchmark schemes, in terms of the error performance. More importantly, we unveil the corresponding relations between the achievable rate in the idealistic case and coded BER in the realistic case, e.g., with finite alphabet input, for the RS precoded multicarrier multigroup multicast scenario.
\end{abstract}

Index Terms-Downlink multiuser MISO, multicarrier multigroup multicast, rate-splitting, optimization, coded bit error rate BER.

This work was supported in part by the U.K. Engineering and Physical Sciences Research Council (EPSRC) under Grant EP/P03456X/1, and in part by the 111 Plan of China under Grant BP0719010. The authors would like to acknowledge the support of the University of Surrey 5GIC \& 6GIC members for this work. Part of the material in this paper was presented at the IEEE International Symposium on Personal, Indoor and Mobile Radio Communications (PIMRC) 2020 Virtual Conference [1].

H. Chen, D. Mi, Z. Chu and P. Xiao are with the 5GIC \& 6GIC Institute for Communication Systems (ICS), University of Surrey, Guildford GU2 7XH, U.K. (e-mail: hongzhi.chen@surrey.ac.uk; d.mi@surrey.ac.uk; andrew.chuzheng7@gmail.com; p.xiao@surrey.ac.uk).

$\mathrm{T}$. Wang is with the Information and Communication Engineering College, Harbin Engineering University, Nantong Street 145, Harbin 150001, China (e-mail: wangtong@ hrbeu.edu.cn).

Y. Xu and D. He are with the School of Electronic Information and Electrical Engineering, Shanghai Jiao Tong University, Shanghai 200240, China (e-mail: xuyin@sjtu.edu.cn, hedazhi@sjtu.edu.cn).

\section{INTRODUCTION}

$\mathbf{I}$ $\mathrm{T}$ is evident that 2021, global mobile data traffic will exceed 50 exabytes per month. A considerable portion of this huge amount of data comes from consuming of common content, e.g., live broadcast of sporting events, breaking news notification, and software/firmware update. How to efficiently deliver the common content motivates a plethora of technological innovations. For instance, the 3rd Generation Partnership Project (3GPP) introduced the multimedia broadcast/multicast service (MBMS) in the third and fourth generations of cellular networks, e.g., Long-Term Evolution (LTE) evolved MBMS (eMBMS) [2], [3] which is subsequently enhanced for the fifth generation (5G) in Release 16 [4]. A new work item is approved in 3GPP Release 17 to carry out research and development on 5G New Radio based broadcast/multicast services [5].

In research communities, the topic of physical layer multicast (PHY multicast) has attracted revived attention, i.e., single-group multicast that initially proposed in the Ph.D. thesis of Lopez [6], which introduces a transmit precoder design for the common content delivery. The design strategy of a PHY multicast precoder is to achieve a targeted service requirement such as quality of service (QoS), with certain constraints. Typical examples are maximizing the minimum received signal-to-noise ratio (SNR) subject to the total power constraint [7] or minimizing the transmit power subject to the minimum received SNR value among all users [8]. Closedform precoder results are provided in [9]-[11] for the examples mentioned above, but for special cases, e.g., with a limited number of users or transmit antennas. [9] and [10] provide the closed-form optimal precoder in a two-user multicast scenario, while [11] presents the closed-form SNR region for a two transmit antenna case.

Authors in [12]-[14] investigate more generalised scenarios where there are more than one multicast group. Specifically, a simultaneous transmission of multiple messages to multiple co-channel groups is considered. In the scenario named multigroup multicast (MGMC), the precoding design becomes more relevant compared to the previous single-group multicast scenario, due to the need of addressing a newly introduced intergroup interference (IGI). In [12], [13], the authors formulate the precoder design problem as a quadratically constrained quadratic program (QCQP). Using semi-definite relaxation (SDR), these non-convex QCQP problems are reduced to convex semi-definite programming (SDP) problems. Such problem reformulation/relaxation is likely to disregard the rank-1 
constraint, and thus the acquisition of the optimal precoder vector/matrix requires a rank-1 approximation, e.g., Gaussian approximation [15], [16]. Low complexity algorithms are later proposed, e.g., in [14], [17], which yields a better performance in terms of the percentage of feasible solutions and the total transmitted power compared to that in [13], especially with a relatively large number of users (say more than ten users) [14]. In [17], the author provides an alternative approach to solving the MGMC QoS problem based on a coordinate descent method. However, the approach in [17] requires the system to have as many, if not more than, transmit antennas as the total receiver antennas. It is proved in [18], [19] that with an insufficient number of transmit antennas, all the aforementioned traditional precoding strategies that treat IGI as the noise could result in a relatively small rate growth, especially in the high SNR regime. Inevitably, the number of transmit antennas is very likely to be insufficient in real-world multicast applications. One example could be the multicast for software or firmware updates to a massive number of machinetype devices [3]. In [20], user scheduling is employed to deal with a large pool of receivers, owning an increasing cost of radio resources. Without relying on any scheduling techniques, a rate-splitting (RS) based precoding strategy is developed in [18], [19] to overcome the system performance degradation caused by antenna insufficiency as mentioned above.

Recent studies in [21], [22] regard RS as a general transmission framework that accommodates space-division multiple access (SDMA) and non-orthogonal multiple access (NOMA) as two extreme cases. In contrast to SDMA that fully treats interference as noise and NOMA that fully decodes interference, RS is able to partially decode the interference and partially treat the interference as noise, thus seamlessly bridges these two extreme cases and potentially outperforms them in terms of rate and computational complexity. The majority of works in the field of the RS precoding design for rate optimization can be classified into two categories. In the first category, e.g. [23], the optimal RS precoder is obtained based on the formulated sum-rate maximization or weighted sum-rate maximization problem in a multiuser environment. However, it is indicated in [22] that in the RS-based sum-rate optimization, certain users rate would be forced to zero for the system to reach the maximum sum-rate. Therefore, a QoS threshold per user is considered as an additional constraint in [21], [22], [24], whilst finding such a threshold for different channels can be difficult in practice. In the second category, e.g., in [19], [25], [26], the RS precoder design involves maxmin fairness (MMF) optimization, which achieves a balanced user experience with any given channel. RS has also been applied to an overloaded single-carrier multigroup multicast system [19] and it helps improving the MMF rate compare to other non-orthogonal transmission strategies. A system is defined as overloaded if the number of transmitted streams exceeding the number of spatial Degrees of Freedom (DoF) created through classical precoding. With such scenario, the channel does not have enough null space to suspend all the co-channel interference. Despite the promising performance of RS in overloaded MGMC scenarios, there remain significant research gaps on implementing RS into multicarrier communi- cation environment, and the flexibility of resource allocation or multiuser/multigroup diversity offered by the multicarrier environment is not well-exploited. Taking a traditional multicarrier system as an example, e.g., orthogonal frequency division multiple access (OFDMA) [27], [28], allocating at most one group on each subcarrier to avoid IGI leads to a poor spectral efficiency. Conversely, having too many groups co-located on the same subcarrier considerably increases the IGI, even with adequately designed precoding schemes [29], [30].

This work aims to fill the research gap on the practical implementation of the RS schemes, especially in the overloaded multicarrier MGMC downlink scenario. To this end, we model the system as a multiuser multiple-input single-output (MISO) system aided by the RS based precoder, for which we derive the closed-form optimized precoder matrix and integrate it into a transceiver framework established in this work, and then we evaluate the coded bit error rate (BER) performance via linklevel simulations (LLS). To acquire the optimized RS precoder, a joint max-min fairness and sum subcarrier rate optimization problem is formulated for the considered system. Unlike most of the related works, our problem formulation combines the optimization of the RS precoder, subcarrier allocation and power allocation altogether in a unified framework. The established transceiver chain consists of low-density paritycheck (LDPC) channel encoder, modulator and the optimized RS precoder at the transmitter, an adapted soft-in soft-out based maximum a posteriori (MAP) detector, LDPC decoder and successive interference cancellation (SIC) at the receiver. Simulation results not only verify the effectiveness of the proposed RS precoding scheme compared with benchmark schemes, but also help us gain deep insights into its performance behaviour in practical scenarios, e.g., without sharing the constellation information between users/groups, RS can still effectively decode the information bits in certain streams. We also discuss how the rate performance of individual symbol streams of RS matches with its corresponding coded BER performance. As the former (i.e., the achievable rate) is in general obtained under idealistic assumptions such as Gaussian inputs and infinite code block length, and the latter (i.e., the coded BER) is calculated under more practical assumptions such as finite alphabet inputs and finite block length, the findings in this paper demonstrate the relations between the performances of RS in ideal cases and in realistic cases, which unveils the fact that the performance gain of RS over benchmark schemes in the considered scenario is practically achievable.

The paper is organized as follows. Section II introduces the multicarrier multigroup multicast system under study. Section III delivers the methodology to acquire the optimized RS precoder by solving the formulated optimization problem, via a proposed two-layer WMMSE method. Section IV carries out the transceiver design of the RS system which utilizes the optimized RS precoder. The corresponding simulation results for both rate and error performances are examined in Section V. Finally, conclusions are drawn in Section VI.

Notations: we use lower case letters, boldface lower case letters and boldface upper case letters to denote scalars, vectors 


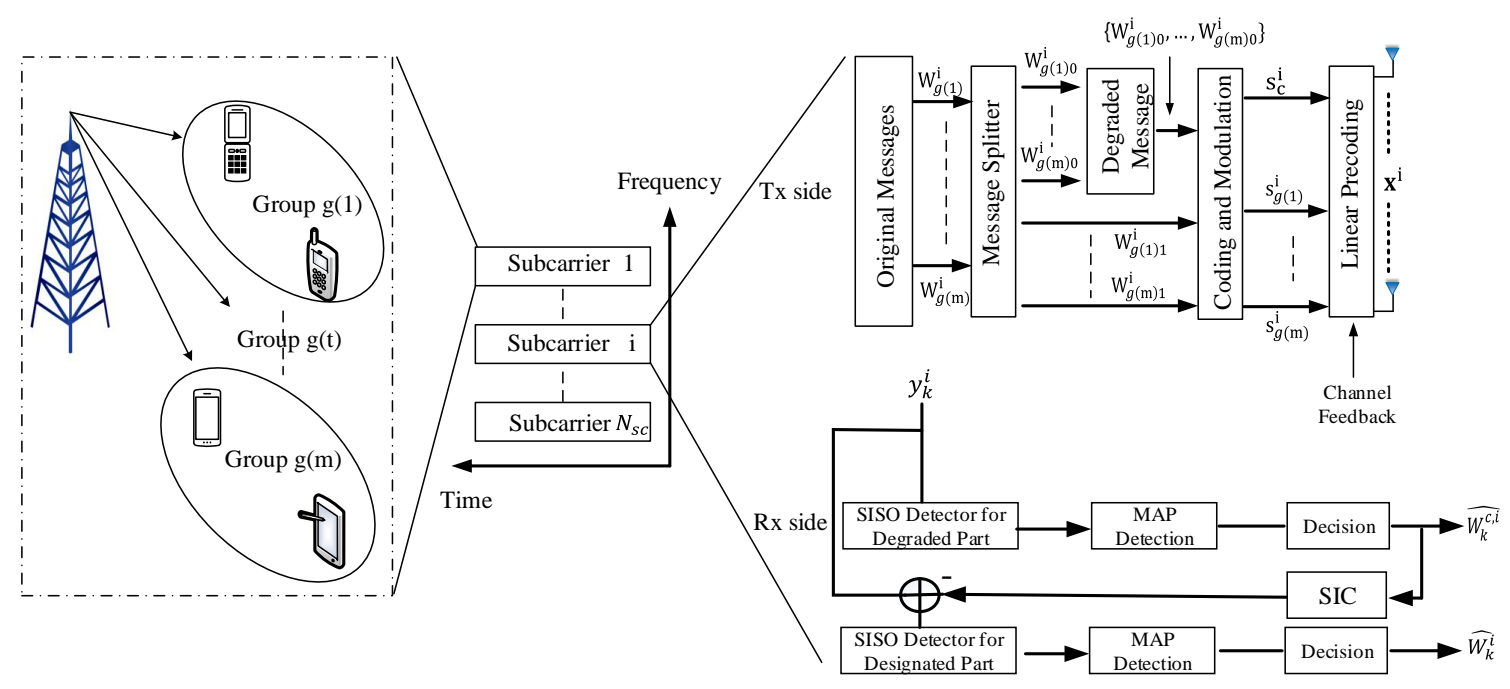

Fig. 1: System model of the RS-SIC transceiver design for the multicarrier multigroup multicast scenario

and matrices respectively. Blackboard bold letters are used to denote sets, $\mathbb{C}$ denotes the set of complex numbers, $\mathbb{C}^{N \times 1}$ denotes the set of $N \times 1$ vectors with complex entries. The superscripts $(\cdot)^{\mathrm{T}}$ and $(\cdot)^{\mathrm{H}}$ denote the transpose and conjugatetranspose operation respectively. $\|\cdot\|, \operatorname{tr}(\cdot)$ and $E\{\cdot\}$ are the second order norm, trace and statistical expectation operators respectively. $\nabla_{x} f(x)$ denotes the partial derivatives of function $f(x)$, comprised by the gradient vector of this function. Calligraphic letters are for sets and notation $\mathbf{X} \succeq 0$ means that $\mathbf{X}$ is a positive semi-definite matrix.

\section{SySTEM MODEL}

Consider a multicast downlink system where a base station (BS) equipped with $N_{t}$ transmit antennas serves $K$ singleantenna users. Such system is often assumed to be overloaded in practice, i.e., $K>N_{t}$. The users, denoted by a set $\mathbb{K} \triangleq\{1, \ldots, K\}$, are grouped into $M$ multicast groups, denoted by a set $\mathbb{M} \triangleq\{1, \ldots, M\}$. We consider an equal user grouping, and that one user can not belong to more than one group at one transmission time. The total available spectrum is equally divided into $N_{\text {sc }}$ orthogonal subcarriers with bandwidth of $B \mathrm{~Hz}$, denoted by a set $\mathbb{N}_{s c} \triangleq\left\{1, \ldots, N_{s c}\right\}$. Each subcarrier can be allocated to $m$ multicast groups, where $1<m \leq M$. We further define subsets $\mathbb{M}^{i} \subseteq \mathbb{M}$ and $\mathbb{K}^{i} \subseteq \mathbb{K}$ as the selected $m$ groups and the corresponding users on the $i$-th subcarrier, respectively. Note that the optimization of the value $m$ is not within the scope of this paper, and we will traverse in system performance evaluations with all possible values of $m$ for a given $M$. The received signal at user $k \in \mathbb{K}^{i}$ is expressed as:

$$
y_{k}^{i}=\left(\mathbf{h}_{k}^{i}\right)^{\mathrm{H}} \mathbf{x}^{i}+n_{k}^{i},
$$

where, for a given $i$-th subcarrier, $\mathbf{x}^{i}, \mathbf{h}_{k}^{i} \in \mathbb{C}^{N_{t}}$ represent the transmitted signal and the channel between the BS and the $k$ th user respectively, whilst $n_{k}^{i} \sim C N\left(0,\left(\sigma_{n, k}^{i}\right)^{2}\right)$ denotes the complex additive white Gaussian noise. Without loss of generality, we assume $\left(\sigma_{n, k}^{i}\right)^{2}=\sigma_{n}^{2}, \forall i, k$. It is further assumed that the channel state information (CSI) is available, e.g., through uplink training in time division duplex or quantized feedback in frequency division duplex systems [23].

We define a mapping function $g(t)$ where $t$ is the index for the allocated $m$ groups on $i$-th subcarrier, such that group messages $W_{g(t)}^{i}(t=1, \ldots, m)$ are delivered to corresponding users in group $g(t)$. The state-of-the-art transmission scheme to deliver multiple group messages, e.g., in LTE-based terrestrial broadcast [3], is time division multiplexing (TDM), equivalents to a single-stream (SS) transmission approach as in [18], where all group messages are packed into one single data stream and broadcast to all groups. Different groups decode their specific group message in different time slots. The main drawback of these orthogonal or SS-like approaches is the under-utilization of available radio resources. We consider to use a non-orthogonal RS-based approach [19], where each message is split into two parts, namely degraded and designated parts, denoted as $W_{g(t)}^{i} \rightarrow\left\{W_{g(t) 0}^{i}, W_{g(t) 1}^{i}\right\}$. All the degraded parts are encoded and modulated into one degraded symbol stream, i.e., $\left\{W_{g(1) 0}^{i}, \ldots, W_{g(t) 0}^{i}, \ldots, W_{g(m) 0}^{i}\right\} \rightarrow s_{c}^{i}$. The designated part of the group message is independently encoded and modulated into $g(m)$ symbol streams, e.g., $W_{g(t) 1}^{i} \rightarrow s_{g(t)}^{i}$. Hence, the transmitted signal on the $i$-th subcarrier in the RS-based system can be given as:

$$
\mathbf{x}^{i}=\mathbf{p}_{c}^{i} s_{c}^{i}+\sum_{t=1}^{m} \mathbf{p}_{g(t)}^{i} s_{g(t)}^{i},
$$

where $\mathbf{p}_{c}^{i}, \mathbf{p}_{g(t)}^{i} \in \mathbb{C}^{N_{t}}$ represent precoding vectors on the $i$ th subcarrier for the degraded symbol stream and designated symbol stream of the $g(t)$-th group, respectively. The power constraint follows $\sum_{i=1}^{N_{s c}} \mathrm{E}\left\{\left(\mathbf{x}^{i}\right)^{H}\left(\mathbf{x}^{i}\right)\right\} \leq \sum_{i=1}^{N_{s c}} P_{i}$, where $P_{i} \geq 0$ is the power allocated on the $i$-th subcarrier. We define 
the total available power as $P \triangleq\left(\sum_{i=1}^{N_{s c}} P_{i}\right)$. The received signal at user $k \in \mathbb{K}^{i}$ in (1) can be rewritten as:

$$
\begin{aligned}
y_{k}^{i} & =\underbrace{\left(\mathbf{h}_{k}^{i}\right)^{\mathrm{H}} \mathbf{p}_{c}^{i} s_{c}^{i}}_{\text {degraded symbol }}+\underbrace{\left(\mathbf{h}_{k}^{i}\right)^{\mathrm{H}} \mathbf{p}_{\mu(k)}^{i} s_{\mu(k)}^{i}}_{\text {designated symbol }} \\
+ & \underbrace{\sum_{t=1, g(t) \neq \mu(k)}^{m}\left(\mathbf{h}_{k}^{i}\right)^{\mathrm{H}} \mathbf{p}_{g(t)}^{i} s_{g(t)}^{i}+n_{k}^{i}}_{\text {IGI+noise }},
\end{aligned}
$$

where $\mu(k)$ is a user-to-group mapping function, meaning that the $k$-th user belongs to the group $\mu(k)$ on the $i$-th subcarrier. The considered multicarrier multigroup multicast system model, along with the RS-based transmitter (Tx) side and receiver (Rx) side, is depicted in Fig. 1. More details on the transceiver design will be given in Section IV.

\section{RS PRECODER ACQUISITION}

\section{A. Problem Formulation}

The concept of 1-layer RS and multi-layer (or so-called generalized) RS is introduced in [24]. The difference mainly comes from the receiver processing, i.e., single layer vs. multiple layers of SIC. Results in [24] show that the lowcomplexity 1-layer RS has a comparable rate performance to the multi-layer RS. The advantage of complexity reduction associated with the 1-layer SIC RS becomes more significant when the number of users increases. In this work, we consider the 1-layer SIC RS based system, where each user first decodes the degraded part, then removes it through successive interference cancellation, and finally decodes the designated part, as elaborated in the following phases:

1) Decoding degraded part: in this phase, the signal-tointerference-plus-noise ratio (SINR) experienced by the $k$-th user $\left(k \in \mathbb{K}^{i}\right)$ can be expressed as:

$$
\eta_{k}^{c, i}=\frac{\left|\left(\mathbf{h}_{k}^{i}\right)^{H} \mathbf{p}_{c}^{i}\right|^{2}}{\sum_{t=1}^{m}\left|\left(\mathbf{h}_{k}^{i}\right)^{H} \mathbf{p}_{g(t)}^{i}\right|^{2}+\sigma_{n}^{2}},
$$

leading to the achievable rate of the degraded symbol stream for the $k$-th user on the $i$-th subcarrier: $R_{k}^{c, i}=B \log _{2}\left(1+\eta_{k}^{c, i}\right)$. Given that every user has to first successfully decode the degraded stream by treating all designated streams as noise, the maximum transmission rate of the degraded symbol stream, denoted as $R^{c, i}$, should meet the following requirement: $R^{c, i}=\min _{k \in \mathbb{K}^{i}} R_{k}^{c, i}$.

2) Decoding designated part: after successfully decoding and subtracting the degraded part from the received signal, each user decodes their corresponding designated part. The received SINR of the designated stream for the $k$-th user is expressed as:

$$
\eta_{k}^{p, i}=\frac{\left|\left(\mathbf{h}_{k}^{i}\right)^{H} \mathbf{p}_{\mu(k)}^{i}\right|^{2}}{\sum_{t=1, g(t) \neq \mu(k)}\left|\left(\mathbf{h}_{k}^{i}\right)^{H} \mathbf{p}_{g(t)}^{i}\right|^{2}+\sigma_{n}^{2}} .
$$

The achievable rate of the designated symbol stream for the $k$-th user on the $i$-th subcarrier is given by: $R_{k}^{p, i}=B \log _{2}(1+$ $\left.\eta_{k}^{p, i}\right)$. Considering that the overall quality of user experience of such a multicast system is strongly dependent on the lowest rate among all involved users, the group rate of the designated stream for the $\mu(k)$-th group on the $i$-th subcarrier is given by: $R_{\mu(k)}^{p, i}=\min _{\forall k \in \mu(k)} R_{k}^{p, i}$. As indicated in the system model, each group contributes a fraction of their original message to form the degraded symbol stream that is intended for all groups. We define:

$$
C_{\mu(k)}^{i}=\frac{\mathbb{L}\left(W_{\mu(k) 0}^{i}\right)}{\sum_{t=1}^{m} \mathbb{L}\left(W_{g(t) 0}^{i}\right)} R^{c, i}
$$

to calculate the rate contribution from the $\mu(k)$-th group, where $\mathbb{L}(\cdot)$ denotes the length of message, and $0 \leq$ $\frac{\mathbb{L}\left(W_{\mu(k) 0}^{i}\right)}{\sum_{t=1}^{m} \mathbb{L}\left(W_{g(t) 0}^{i}\right)} \leq 1$ is the splitting ratio. Therefore, the overall rate for the group $\mu(k)$ on the $i$-th subcarrier is given by: $R_{\mu(k)}^{i}=C_{\mu(k)}^{i}+R_{\mu(k)}^{p, i}$.

\section{B. Optimization Problem Formulation}

In this paper, we consider the max-min fairness optimization on each subcarrier since other optimization strategies such as sum-rate maximization or average-rate maximization do not help improve the lowest group rate. Recall that $m$ out of $M$ groups are allocated on each subcarrier, we take into account different group for subcarrier allocations, and express the MMF rate on the $i$-th subcarrier as:

$$
z^{i}=\left\{\sum_{j=1}^{J} a_{j}^{i}\left(\max _{\mathbf{p}^{i}, \mathbf{C}^{i}} \min _{\mu(k) \in \mathbb{M}^{i}} R_{\mu(k)}^{i}\right) \mid \mathbf{a}, m\right\},
$$

where $\mathbf{p}^{i}=\left[\mathbf{p}_{c}^{i}, \mathbf{p}_{g(1)}^{i}, \ldots, \mathbf{p}_{g(m)}^{i}\right]$ denotes the concatenated precoding vector, and $\mathbf{C}=\left[C_{1}^{i}, \ldots, C_{m}^{i}\right]$ includes the corresponding degraded stream rate of each group. We denote an indicator vector for the $i$-th subcarrier by a column vector $\mathbf{a}_{\mathbf{J}}^{i}$ with dimension of $[J \times 1]$, and $J=\left(\begin{array}{c}M \\ m\end{array}\right)$ is the number of possible group combinations, then we have $\mathbf{J} \triangleq[1, \ldots, J]$. Individual entry of $\mathbf{a}_{\mathbf{J}}^{i}$ is denoted as $a_{j}^{i}, j \in\{1, \ldots, J\}$, where $a_{j}^{i}=1$ means that the $j$-th group allocation combination is applied on the $i$-th subcarriers. $\mathbf{a} \triangleq\left[\mathbf{a}_{\mathbf{J}}^{1}, \ldots, \mathbf{a}_{\mathbf{J}}^{N_{s c}}\right]$ denotes the collection of indicator vectors on all subcarrier. Given (7) and $P=\left(\sum_{i=1}^{N_{s c}} P_{i}\right)$, we can formulate an optimization problem for the RS-based joint subcarrier allocation and transmit precoder design problem, as follows:

$$
\begin{aligned}
\max _{\mathbf{p}^{i}, \mathbf{a}, \mathbf{C}, \mathbf{z}} & \sum_{i=1}^{N_{s c}} z^{i}, \\
\text { s.t. } & a_{j}^{i} \in\{0,1\}, \forall j, i \\
& \sum_{j=1}^{J} a_{j}^{i} \leq 1, \forall i \\
& \sum_{j=1}^{J} \sum_{i=1}^{N_{s c}} a_{j}^{i}\left(\left\|\mathbf{p}_{c}^{i}\right\|^{2}+\sum_{t=1}^{m}\left\|\mathbf{p}_{g(t)}^{i}\right\|^{2}\right) \leq P \\
& \sum_{t=1}^{m} C_{g(t)}^{i}=R^{c, i}, C_{g(t)}^{i} \geq 0 \\
& C_{\mu(k)}^{i}+R_{\mu(k)}^{p, i} \geq z^{i}, \forall k \in \mathbb{K}^{i} \\
& R_{k}^{c, i} \geq R^{c, i}, \forall k \in \mathbb{K}^{i}
\end{aligned}
$$


where $\mathbf{z} \triangleq\left[z^{1}, \ldots, z^{N_{s c}}\right]$, with $z^{i}$ denoting the MMF rate on the $i$-th subcarrier. The conditions in (8b) and (8c) are to restrict the group allocation on each subcarrier, in (8d) for the transmission power restriction, and in $(8 \mathrm{e})$ to guarantee the decodability of the degraded part. The optimization problem (8) is non-convex, and thus the existing methods like semidefinite relaxation (SDR) in [31] and [32] cannot be used. In Section III-C, this problem is solved by exploiting the relationship between the achievable rate and weighted minimum mean square error (WMMSE).

\section{Solutions to the Optimization Problem}

In this section, we solve problem (8) using the WMMSE based optimization which leads to a two layer optimization algorithm.

1) Received signal power reformulation: Taking into account the subcarrier allocation indicator, based on (2) and (3), the received signal for the $k$-th user $\left(k \in \mathbb{K}^{i}\right)$ is given as:

$$
\begin{aligned}
y_{k}^{i} & =\left(\mathbf{h}_{k}^{i}\right)^{H} \mathbf{x}^{i}+n_{k} \\
& =\left(\mathbf{h}_{k}^{i}\right)^{H}\left(\sum _ { j = 1 } ^ { J } a _ { j } ^ { i } \left(\mathbf{p}_{c}^{i} s_{c}^{i}+\mathbf{p}_{\mu(k)}^{i} s_{\mu(k)}^{i}\right.\right. \\
& \left.\left.+\sum_{t=1, g(t) \neq \mu(k)}^{m}\left(\mathbf{h}_{k}^{i}\right)^{\mathrm{H}} \mathbf{p}_{g(t)}^{i} s_{g(t)}^{i}+n_{k}^{i}\right)\right) .
\end{aligned}
$$

Since $a_{j}^{i}=\{0,1\}$, we have $\sum_{j=1}^{J} a_{j}^{i}\left(a_{j}^{i}\right)^{H}=\sum_{j=1}^{J} a_{j}^{i}$. The received power can be calculated as $T_{k}^{i}=\mathbf{E}\left\{y_{k}^{i}\left(y_{k}^{i}\right)^{H}\right\}$, i.e.,

$$
\begin{aligned}
T_{k}^{i} & =\underbrace{\sum_{j=1}^{J} a_{j}^{i}\left(\mathbf{h}_{k}^{i}\right)^{H} \mathbf{p}_{c}^{i}\left(\mathbf{p}_{c}^{i}\right)^{H} \mathbf{h}_{k}^{i}}_{\text {degraded symbol power }}+ \\
& \underbrace{\sum_{j=1}^{J} a_{j}^{i}\left(\mathbf{h}_{k}^{i}\right)^{H} \mathbf{p}_{\mu(k)}^{i}\left(\mathbf{p}_{\mu(k)}^{i}\right)^{H} \mathbf{h}_{k}^{i}}_{\text {designated symbol power }} \\
& +\underbrace{\sum_{j=1}^{J} \sum_{t=1, g(t) \neq \mu(k)}^{m} a_{j}^{i}\left(\mathbf{h}_{k}^{i}\right)^{H} \mathbf{p}_{g(t)}^{i}}_{\text {IGI+noise power }}\left(\mathbf{p}_{g(t)}^{i}\right)^{H} \mathbf{h}_{k}^{i}+\sigma_{n}^{2} \\
& =S_{k}^{c, i}+S_{k}^{i}+I_{k}^{i},
\end{aligned}
$$

where $S_{k}^{c, i}, S_{k}^{i}$, and $I_{k}^{i}$ denote the power as shown in the corresponding under brackets, respectively. The SINRs can be represented as: $\eta_{k}^{c, i}=\frac{S_{k}^{c, i}}{S_{k}^{i}+I_{k}^{i}}$ and $\eta_{k}^{p, i}=\frac{S_{k}^{i}}{I_{k}^{i}}$.

2) Formulating the relationship between achievable rate and MMSE: At the receiver, we consider the minimum mean square error (MMSE) based equalizer design which is intended to minimize:

$$
\begin{aligned}
& \epsilon_{k}^{c, i} \triangleq \mathbf{E}\left\{\left|\widehat{s_{k}^{c, i}}-\sum_{j=1}^{J} a_{j}^{i} s_{c}^{i}\right|^{2}\right\}, k \in \mathbb{K}^{i}, \forall i \in \mathbb{N}_{s c} \\
& \epsilon_{k}^{i} \triangleq \mathbf{E}\left\{\left|\widehat{s_{k}^{i}}-\sum_{j=1}^{J} a_{j}^{i} s_{\mu(k)}^{i}\right|^{2}\right\}, k \in \mathbb{K}^{i}, \forall i \in \mathbb{N}_{s c}
\end{aligned}
$$

where $\epsilon_{k}^{c, i}$ and $\epsilon_{k}^{i}$ are the mean square error (MSE) of the estimated degraded (i.e. $\widehat{s_{c, k}^{i}}$ ) and designated symbol (i.e. $\widehat{s_{k}^{i}}$ ) by the $k$-th user, on the $i$-th subcarrier. The corresponding estimated symbols are calculated by: $\widehat{s_{c, k}^{i}}=g_{k}^{c, i} y_{k}^{i}$, and $\widehat{s_{k}^{i}}=$ $g_{k}^{i}\left(y_{k}^{i}-\left(\mathbf{h}_{k}^{i}\right)^{H} \mathbf{p}_{c}^{i} \widehat{s_{c, k}^{i}}\right)$, where $g_{k}^{c, i}$ and $g_{r}^{i}$ denote the equalizers that functioned on the received signal, before and after SIC, respectively. Therefore, starting with the degraded symbol, by expanding (11) we have:

$$
\begin{aligned}
& \mathbf{E}\left\{\left|\widehat{s_{k}^{c, i}}-\sum_{j=1}^{J} a_{j}^{i} s_{c}^{i}\right|^{2}\right\} \\
& =\left|g_{k}^{c, i}\right|^{2} \mathbf{E}\left\{y_{k}^{i}\left(y_{k}^{i}\right)^{H}\right\}-\mathbf{E}\left\{\sum_{j=1}^{J} a_{j}^{i} g_{k}^{c, i} y_{k}^{i}\left(s_{c}^{i}\right)^{H}\right\} \\
& -\mathbf{E}\left\{\sum_{j=1}^{J} a_{j}^{i} s_{c}^{i}\left(y_{k}^{i}\right)^{H}\left(g_{k}^{c, i}\right)^{H}\right\}+\sum_{j=1}^{J}\left(a_{j}^{i}\right)^{2} .
\end{aligned}
$$

Since $\mathbf{E}\left\{s_{\mu(k)}^{i}\left(s_{c}^{i}\right)^{H}\right\}=0, \mathbf{E}\left\{s_{g(t)}^{i}\left(s_{c}^{i}\right)^{H}\right\}=0$ and $\mathbf{E}\left\{n_{k}^{i}\left(s_{c}^{i}\right)^{H}\right\}=0$, further expanding $y_{k}^{i}$ yields:

$$
\begin{aligned}
& \mathbf{E}\left\{\left|\widehat{s_{k}^{c, i}}-\sum_{j=1}^{J} a_{j}^{i} s_{c}^{i}\right|^{2}\right\} \\
& =\left|g_{k}^{c, i}\right|^{2} T_{k}^{i}-2 \Re \mathfrak{R e}\left\{\sum_{j=1}^{J} a_{j}^{i} g_{k}^{c, i}\left(\mathbf{h}_{k}^{i}\right)^{H} \mathbf{p}_{c}^{i}\right\}+1 .
\end{aligned}
$$

Here we define $\mathfrak{R e}\{\cdot\}$ as the real value operation, and $\operatorname{Im}\{\cdot\}$ as the imagination value operation. For any given value of subcarrier indicator matrix a, the equalizer coefficient $g_{k}^{c, i}$ is calculated as:

$$
\begin{aligned}
& \nabla_{g_{k}^{c, i}} \mathbf{E}\left\{\left|\widehat{s_{k}^{c, i}}-\sum_{j=1}^{J} a_{j}^{i} s_{c}^{i}\right|^{2}\right\}=0 \\
& \Rightarrow 2 g_{k}^{c, i} T_{k}^{i}-2 \mathfrak{R e}\left\{\sum_{j=1}^{J} a_{j}^{i}\left(\mathbf{p}_{c}^{i}\right)^{H} \mathbf{h}_{k}^{i}\right\}=0 \\
& \Rightarrow g_{k}^{c, i}=\frac{\mathfrak{R e}\left\{\sum_{j=1}^{J} a_{j}^{i}\left(\mathbf{p}_{c}^{i}\right)^{H} \mathbf{h}_{k}^{i}\right\}}{T_{k}^{i}} .
\end{aligned}
$$

Therefore, we can drop the constraints (8b) and (8c). Substituting (15) back to (14) yields the minimum MSE of the estimated degraded symbol, given as:

$$
\begin{aligned}
\epsilon_{k, \text { min }}^{c, i} & =\frac{\mathfrak{R e}\left\{\left|\sum_{j=1}^{J} a_{j}^{i}\left(\mathbf{p}_{c}^{i}\right)^{H} \mathbf{h}_{k}^{i}\right|^{2}\right\}}{\left(T_{k}^{i}\right)^{2}} T_{k}^{i} \\
& -2 \mathfrak{R e}\left\{\frac{\sum_{j=1}^{J} a_{j}^{i}\left(\mathbf{p}_{c}^{i}\right)^{H}\left(\mathbf{h}_{k}^{i}\right)\left(\mathbf{h}_{k}^{i}\right)^{H} \mathbf{p}_{c}^{i}}{T_{k}^{i}}\right\}+1,
\end{aligned}
$$

since $S_{k}^{c, i}=\sum_{j=1}^{J} a_{j}^{i}\left(\mathbf{h}_{k}^{i}\right)^{H} \mathbf{p}_{c}^{i}\left(\mathbf{p}_{c}^{i}\right)^{H} \mathbf{h}_{k}^{i}$ and $\mathfrak{I m}\left\{S_{c, r}^{i}\right\}=0$, therefore,

$$
\epsilon_{k, \min }^{c, i}=\left(1+\frac{S_{k}^{c, i}}{S_{k}^{i}+I_{k}^{i}}\right)^{-1}=\left(1+\eta_{k}^{c, i}\right)^{-1} .
$$

Thus, the relationship between the MMSE and the achievable rate for the degraded symbol stream can be established, as follows:

$$
R_{k}^{c, i}=\log _{2}\left(1+\eta_{k}^{c, i}\right)=-\log _{2}\left(\epsilon_{k, \text { min }}^{c, i}\right)
$$


The same applies while estimating the MSE of the designated stream, denoted as $\mathbf{E}\left\{\mid g_{k}^{i}\left(y_{k}^{i}-\left(\mathbf{h}_{k}^{i}\right)^{H} \mathbf{p}_{c}^{i} \widehat{s_{c, k}^{i}}\right)-\right.$ $\left.\left.\sum_{j=1}^{J} a_{j}^{i} s_{\mu(k)}^{i}\right|^{2}\right\}$. The corresponding equalizer is:

$$
g_{k}^{i}=\frac{\mathfrak{R e}\left\{\sum_{j=1}^{J} a_{j}^{i}\left(\mathbf{p}_{\mu(k)}^{i}\right)^{H}\left(\mathbf{h}_{k}^{i}\right)\right\}}{S_{r}^{i}+I_{r}^{i}},
$$

and the corresponding MMSE is:

$$
\begin{aligned}
\epsilon_{k, \min }^{i} & =\frac{\mathfrak{R e}\left\{\left|\sum_{j=1}^{J} a_{j}^{i}\left(\mathbf{p}_{\mu(k)}^{i}\right)^{H}\left(\mathbf{h}_{k}^{i}\right)\right|^{2}\right\}}{S_{k}^{i}+I_{k}^{i}} \\
& -2 \mathfrak{R e}\left\{\frac{\sum_{j=1}^{J} a_{j}^{i}\left(\mathbf{p}_{\mu(k)}^{i}\right)^{H}\left(\mathbf{h}_{k}^{i}\right)\left(\mathbf{h}_{k}^{i}\right)^{H} \mathbf{p}_{\mu(k)}^{i}}{S_{k}^{i}+I_{k}^{i}}\right\}+1 \\
& =\frac{\mathfrak{R e}\left\{S_{k}^{i}\right\}}{S_{k}^{i}+I_{k}^{i}}-2 \mathfrak{R e}\left\{\frac{S_{k}^{i}}{S_{k}^{i}+I_{k}^{i}}\right\}+1 \\
& =\left(1+\frac{S_{k}^{i}}{I_{k}^{i}}\right)^{-1}=\left(1+\eta_{k}^{p, i}\right)^{-1} .
\end{aligned}
$$

Thus, the achievable rate for the designated stream is given by: $R_{k}^{p, i}=\log _{2}\left(1+\eta_{k}^{p, i}\right)=-\log _{2}\left(\epsilon_{k, \text { min }}^{i}\right)$.

3) Reformulating the optimization problem with weighted MMSE: To solve the problem in (8), we consider using the weighted-MSE (WMSE) with a proper set of MSE weights [25]. The following proposition and its proof demonstrate an equivalence between the optimization of the degraded/designated symbol stream MMF rates and the optimization of the corresponding WMMSE.

Proposition 1. Focusing on the degraded stream rate, a simplified max-min rate fairness problem can be established as follows:

$$
\begin{aligned}
\max _{\mathbf{p}_{c}, \mathbf{a}} & \min _{k \in \mu(k), \forall i} R_{k}^{c, i} \\
\text { s.t. } & \sum_{j=1}^{J} \sum_{i=1}^{N_{s c}} a_{j}^{i}\left\|\mathbf{p}_{c}^{i}\right\|^{2}=P_{c}^{o p t}
\end{aligned}
$$

where $P_{c}^{o p t}$ denotes the total power allocated to the degraded symbol on all subcarrier. Define $w_{k}^{c, i} \geq 0$ as a weight scalar for the $k$-th user on the $i$-th subcarrier, problem (21) is equivalent to the following min-max problem, with its objective being a function of the MSE and its weight:

$$
\min _{\mathbf{p}_{c}, \mathbf{a}} \max _{k \in \mu(k), \forall i} f\left(w_{k}^{c, i}, \epsilon_{k}^{c, i}\left(g_{k}^{c, i}\right)\right)
$$$$
\text { s.t. (21b) }
$$

where $f\left(w_{k}^{c, i}, \epsilon_{k}^{c, i}\left(g_{k}^{c, i}\right)\right)=-\frac{1}{\ln 2}+\frac{w_{k}^{c, i} \epsilon_{k}^{c, i}}{\ln 2}-\log _{2} w_{k}^{c, i}$ and as we know $\epsilon_{k}^{c, i}$ is a function of $g_{k}^{c, i} \cdot f\left(w_{k}^{c, i}, \epsilon_{k}^{c, i}\left(g_{k}^{c, i}\right)\right)$ is convex in each variable, i.e. $w_{k}^{c, i}, \epsilon_{k}^{c, i}$ and $g_{k}^{c, i}$, when fixing the others.

Proof: By applying the first order optimality conditions to the objective function in (22), the optimal weight scalar, denoted as $w_{k}^{c, i, o p t}$, can be expressed as a function of the corresponding MMSE value i.e., $\epsilon_{k, \text { min }}^{c, i}$ in (15), given as follows:

$$
\left\{\begin{array}{l}
\nabla_{w_{k}^{c, i}} f\left(w_{k}^{c, i}, \epsilon_{k}^{c, i}\left(g_{k}^{c, i}\right)\right), \\
\nabla_{g_{k}^{c, i}} f\left(w_{k}^{c, i}, \epsilon_{k}^{c, i}\left(g_{k}^{c, i}\right)\right),
\end{array} \Rightarrow w_{k}^{c, i, \text { opt }}=\left(\epsilon_{k, \text { min }}^{c, i}\right)^{-1}\right.
$$

Substituting the results in (23) into (22), we have the following optimization problem:

$$
\min _{\mathbf{p}_{c}, \mathbf{a}} \max _{k \in \mu(k), \forall i}-\log _{2}\left(\epsilon_{k, \min }^{c, i}\right)^{-1}
$$

Combining (24) with (18) completes the proof.

Same proposition can be applied to the designated stream. Hence, the constraints in the original optimization problem, i.e., (8f) and (8g), can be reformulated by using the corresponding MMSEs as follows:

$$
\begin{aligned}
\zeta_{k}^{c, i} & =-\frac{1}{\ln 2}+\frac{w_{k}^{c, i} \epsilon_{k}^{c, i}}{\ln 2}-\log _{2} w_{k}^{c, i} \Rightarrow \zeta_{k}^{c, i, \mathrm{MMSE}}=-R_{k}^{c, i} \\
\zeta_{k}^{i} & =-\frac{1}{\ln 2}+\frac{w_{k}^{i} \epsilon_{k}^{i}}{\ln 2}-\log _{2} w_{k}^{i} \Rightarrow \zeta_{k}^{i, \mathrm{MMSE}}=-R_{k}^{i}
\end{aligned}
$$

where $\zeta_{k}^{c, i}$ and $\zeta_{k}^{i}$ denote the WMSE for degraded and designated streams, respectively. $w_{k}^{i} \geq 0$ is the weight scalar for the corresponding designated symbol stream. Hence, the WMSE reformulation of the original optimization problem can be reformulated as:

$$
\begin{aligned}
\max _{\mathbf{p}^{i}, \mathbf{C}, \mathbf{z}, \mathbf{a}, \mathbf{w}, \mathbf{g}} & \sum_{i=1}^{N_{s c}} z^{i}, \\
\text { s.t. } & (8 \mathrm{~d}),(8 \mathrm{e}) \\
& C_{\mu(k)}^{i}-\zeta_{k}^{i} \geq z^{i}, \forall k \in \mathbb{K}^{i} \\
& -\zeta_{k}^{c, i} \geq R^{c, i}, \forall k \in \mathbb{K}^{i},
\end{aligned}
$$

where $\mathbf{w} \triangleq\left\{w_{k}^{c, i}, w_{k}^{i} \mid i \in \mathbb{N}_{s c}, k \in \mathbb{K}^{i}\right\}$ denotes the weights for degraded and designated symbol streams and $\mathrm{g} \triangleq\left\{g_{k}^{c, i}, g_{k}^{i} \mid i \in \mathbb{N}_{s c}, k \in \mathbb{K}^{i}\right\}$ denotes all the equalizers. As stated, we dropped the constraints (8b) and (8c) by giving (27) one of the possible subcarrier indicator matrices, i.e., $\mathbf{a}^{(n)}, n \in\{1, \ldots, N\}$, where $N=J^{N_{s c}}$ denotes the total number of possible subcarrier indicator matrices. Therefore, with the WMMSE method, we have to search for the best subcarrier allocation indicator matrix by comparing the optimization results for the reformulated problem with each given a. More specifically, problem (27) can be solved by employing an alternating optimization (AO) method. At each iteration of the 'inner' AO procedure, with given a, $\mathbf{w}$ and $\mathbf{g}$ are firstly updated for each subcarrier by solving the MMSE equation in (15) and (19) with an initialized precoder matrix $\mathbf{p}$. Then with the updated $\mathbf{w}$ and $\mathbf{g}$, the precoder $\mathbf{p}$ can be updated by solving (27). Then, for the 'outer' layer, it searches inside the total $N$ possible subcarrier indicator matrices and finds the one which gives the highest sum-MMF rate. Thus, the final sum-MMF subcarrier rate is: $\max \left\{\sum_{i=1}^{N_{s c}} z^{i, \mathbf{a}^{(1)}}, \ldots, \sum_{i=1}^{N_{s c}} z^{i, \mathbf{a}} \mathbf{( n )}^{(n)}, \ldots, \sum_{i=1}^{N_{s c}} z^{i, \mathbf{a}^{(N)}}\right\}$, where $\sum_{i=1}^{N_{s c}} z^{i, \mathbf{a}^{(n)}}$ denotes the result by using the $n$-th possible subcarrier indicator matrix. The above-discussed operations are summarized in Algorithm 1.

\section{IMPLEMENTATION DESIGN OF RATE-SPLITTING}

In previous section, we have investigated the RS based joint precoding and subcarrier allocation design under the 


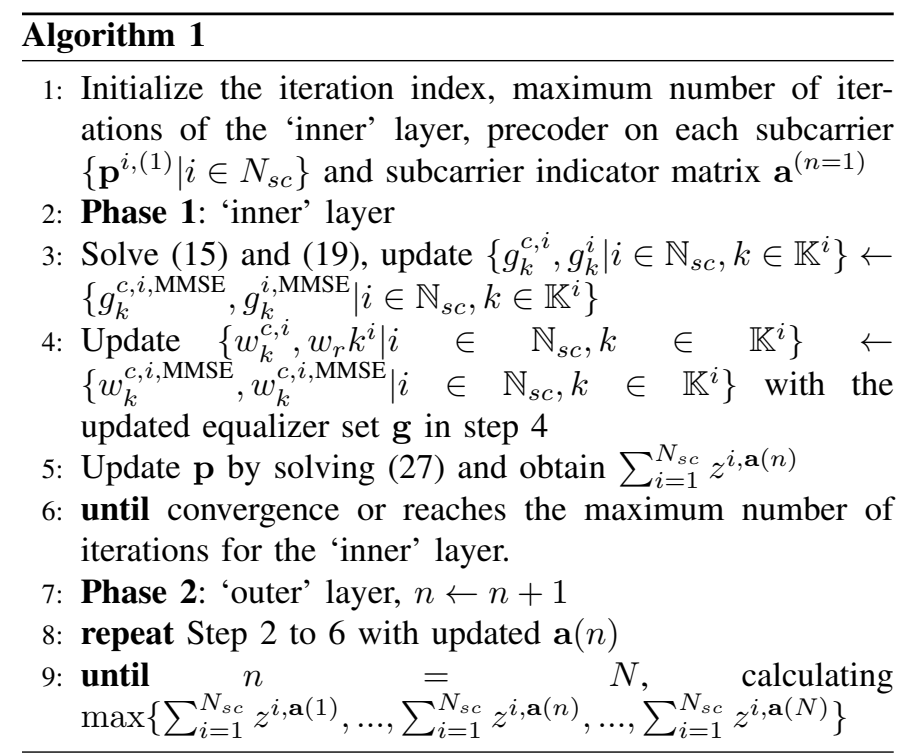

Gaussian signalling. In order to validate the benefit of RS in practical systems and move forwards to its real implementation, here, we take advantage of the optimal precoder matrix provided by the proposed WMMSE method, and establish a transceiver structure and carry out an investigation of the coded bit error rate (BER) with relaxed assumptions such as finite constellation input, finite code block length and specific code rate. Specifically, at the transmitter, channel encoder and the optimal RS precoder are employed for each channel realization. At the receiver, each user uses the same soft-input soft-output (SISO) detection and decoding processes that are adopted to the SIC receiver structure.

\section{A. Transceiver Design}

Fig. 1 already illustrated the transmission block of the RS system under study. Information bits are encoded by the LDPC encoder. This is followed by PSK modulation, before going into the precoder, where the total $M+1$ coded symbol streams are multiplied by the obtained RS precoding matrix from the rate optimization. Finally, all data signals are mapped onto the $N_{t}$ antennas. Despite the fact that the considered system is a multicarrier system, the same transceiver structure applies to each subcarrier, with the obtained optimized subcarrier allocation matrix, we can then transmit specific group messages on specific subcarriers.

1) Precoder, Encoder and Modulator: The employed precoders are the optimization results by solving the MMF problem formulated in the previous section. In addition to the precoder, the obtained MMF rate results can either help us predict the error performance behaviour when we assign different streams the same modulation and coding scheme (MCS) setup, or guide us to design specific MCS schemes for different streams. More specifically, given that the rate optimization results for the degraded and designated stream on one subcarrier are $R_{c}, R_{1}, \ldots, R_{m}$,

- Different MCS for different streams: In this case, one can have the modulation alphabets $\mathbf{M}_{c}, \mathbf{M}_{1}, \ldots, \mathbf{M}_{m}$ and code rates $r_{c}, r_{1}, \ldots, r_{m}$ for the degraded and designated streams, respectively, as shown in [33]. The MCS is selected according to the optimization results in terms of bits per channel use. For instance, $R_{c} \geq \log _{2} \mathbf{M}_{c} * r_{c}$ where the value of $\mathbf{M}_{c}$ and $r_{c}$ are selected from all the possible finite constellation alphabet and LDPC data rate.

- Same MCS for all streams: Two scenarios occur here: (1) The operating bits per channel use exceeds the optimized rate results, consequently, the error performance might degrade significantly. However, the average number of successfully recovered bits per channel use should be the same as the previous MCS setting, for the following reason: within the optimization process, there is no constraint set on the modulation, which means as long as the identity power constraint is met, any modulation scheme can be applied; after rate optimization, the encoder/decoder are expected to have the optimal performance and the achievable rate is fixed at a certain value, e.g. 0.5 bits $/ \mathrm{s} / \mathrm{Hz} @ 5 \mathrm{~dB}$ SNR, which can be achieved by using BPSK with coding rate 0.5 , i.e., $1 * 0.5=0.5 \mathrm{bps}$, or QPSK with code rate 0.25 , i.e., $2 * 0.25=0.5 \mathrm{bps}$. From the throughput perspective, for a given MCS, the throughput can be expressed as $T=R(1-\beta)$, where $\beta$ denotes the corresponding block error rate (BLER). Therefore, the throughput value should still be equivalent to the previous MCS setting rule. (2) The operating bits per channel use are less than the optimized rate results. If those two values are close enough, then it becomes the same as the previous MCS setting rule. Otherwise, the throughput might not be as close as the rate optimization results, but the coded BER should still be able to exhibit the superiority by using RS, under correct/fair MCS setup between RS and No-RS schemes.

In this paper, we are going to follow the second rule, i.e, the same MCS for all streams. Firstly, due to the fact that the considered system works in a multicarrier environment, one group's message might be transmitted over multiple subcarriers, for which they should all use the same modulation alphabet rather than following the rate optimization results. Secondly, the LDPC code has a finite code rate choice which will also affect the code block length and thus make the comparison unfair. For the modulator, we consider the phase shift key (PSK) modulation scheme up to 8PSK. The consecutive encoded bits are mapped to one of the constellation symbols which follows Gray labelling rule. To guarantee fairness between schemes with and without rate-splitting due to the extra symbol stream, we ensure the same amount of bits are transmitted per channel use. For example, without RS the total $M$ streams all employ modulation $\mathbf{M}$, which means the total number of bits per channel user is $M * \log _{2} \mathbf{M}$, therefore we need to have: $M * \log _{2} \mathbf{M}=(M+1) * \log _{2} \hat{\mathbf{M}}$, where $\hat{\mathbf{M}}$ is the modulation level for the RS scheme.

2) Receiver: At the receiver side, inspired by [34], [35], we consider the SISO detector. Among various soft detection methods, the maximum a posteriori detection is selected as it can achieve the optimal performance [36]. In Fig. 2, we illustrate the iterative receiver structure with the SIC process for the $k$-th user. In this work, we consider there is no joint detection and decoding among different group of users. The 


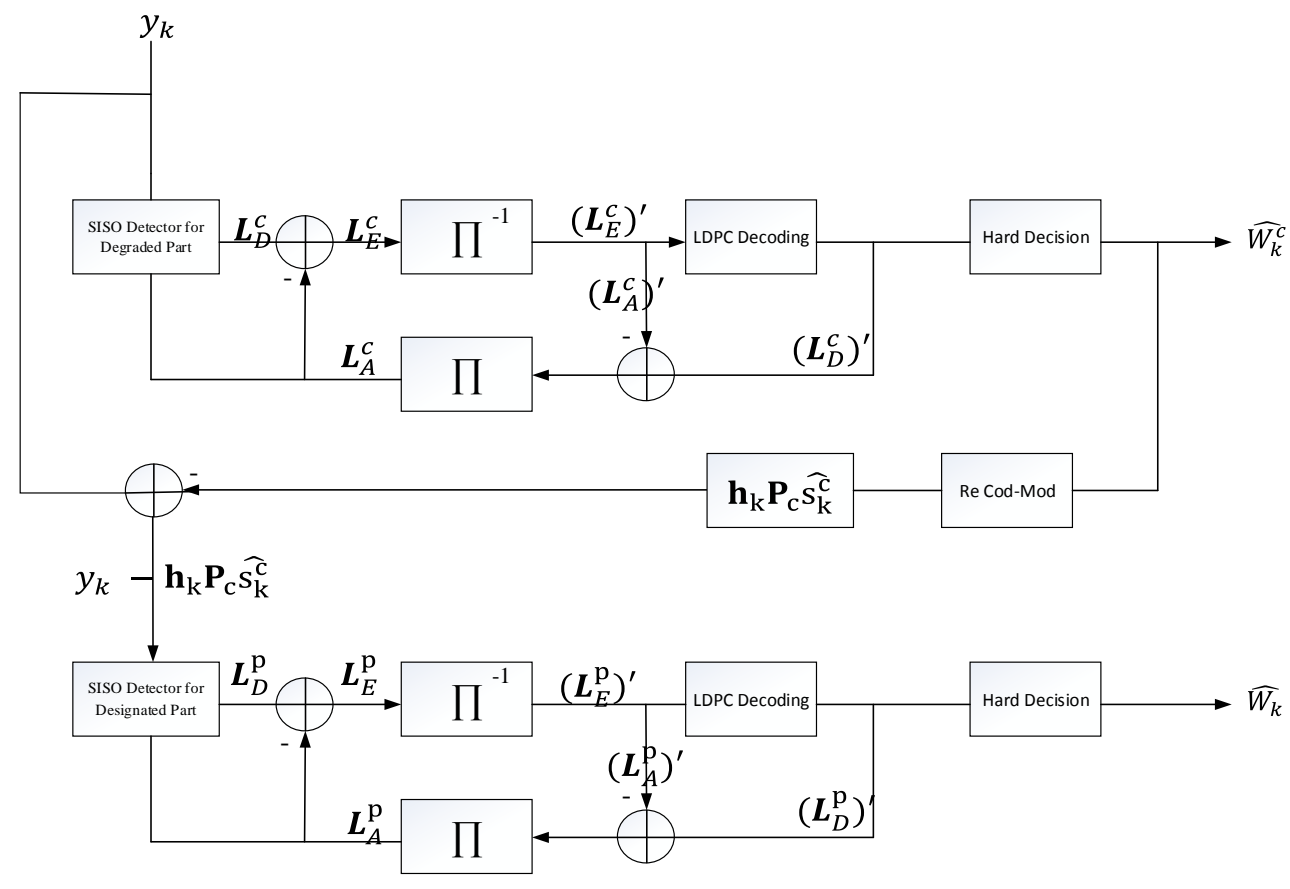

Fig. 2: Iterative receiver diagram for the $k$-th user.

$$
L_{E}\left(b_{k, j_{s}}\right)=\log _{2}\left(\frac{\sum_{j_{s}=1, \mathbf{b}_{k} \in \mathbb{B}_{j_{s},+1}}^{2^{\left(J_{s}-1\right)}} p\left[y_{k} \mid s_{\mu(k)}=\operatorname{map}\left(\mathbf{b}_{k}\right)\right] e^{\frac{1}{2} \mathbf{b}_{k,\left[j_{s}\right]}^{T} \mathbf{L}_{A}\left(\mathbf{b}_{k,\left[j_{s}\right]}\right)}}{\sum_{j_{s}=1, \mathbf{b}_{k} \in \mathbb{B}_{j_{s},-1}}^{2^{\left(J_{s}-1\right)}} p\left[y_{k} \mid s_{\mu(k)}=\operatorname{map}\left(\mathbf{b}_{k}\right)\right] e^{\frac{1}{2} \mathbf{b}_{k,\left[j_{s}\right]}^{T} \mathbf{L}_{A}\left(\mathbf{b}_{k,\left[j_{s}\right]}\right)}}\right)
$$

$$
\begin{aligned}
p\left[y_{k} \mid s_{c}\right. & \left.=\operatorname{map}\left(\mathbf{b}_{c}\right)\right]=\frac{1}{2^{J_{s}} \pi \sigma^{2}} \sum_{i_{s}=1}^{2^{J_{s}}} e^{-\frac{\left\|y_{k}-\mathbf{h}_{k}^{H} \mathbf{P}_{c} s_{c}-\mathbf{P}_{\mu(k)} s_{i s}\right\|^{2}}{\sigma^{2}}} \\
p\left[y_{k} \mid s_{\mu(k)}=\operatorname{map}\left(\mathbf{b}_{k}\right)\right] & =\frac{1}{\pi \sigma^{2}} e^{-\frac{\left\|y_{k}-\mathbf{h}_{k}^{H} \mathbf{P}_{c} \widehat{s}_{c}-\mathbf{h}_{k}^{H} \mathbf{P}_{\mu(k)} s_{\mu(k)}\right\|^{2}}{\sigma^{2}}}
\end{aligned}
$$

system model under study is a downlink multigroup multicast system, so users in one group do not have knowledge of other groups multicast stream information (constellation diagram) and can only trade the other multicast streams as interference when decoding. One exception is for the degraded stream since it should be decoded by all groups of users, thus its constellation information is available to all users. Therefore, each user's knowledge about the constellation is summarised as follows:

- RS scheme: The $k$-th only knows the constellation information of the degraded symbol as well as the specific multicast stream for the $\mu(k)$-th group.

- No-RS scheme: The $k$-th only knows the constellation information for the specific multicast stream for the $\mu(k)$ th group.

For the MAP decoding process, when the $k$-th user receives signal $y_{k}$, it first decodes the degraded part. The SISO detector calculates the extrinsic information of the degraded part, i.e., $\mathbf{L}_{E}^{c}$, based on a priori information $\mathbf{L}_{A}^{c}$. The output $\log$ likelihood ratio (LLR), i.e., $\left(\mathbf{L}_{E}^{c}\right)^{\prime}$, is then interleaved and fed into the LDPC decoder. The LDPC decoder generates the intrinsic information, i.e., $\left(\mathbf{L}_{D}^{c}\right)^{\prime}$, which, upon subtracting the previous intrinsic information, becomes a new a priori information $\mathbf{L}_{A}^{c}$ for the next iteration. In the final iteration, the LDPC decoder outputs only the soft information of the useful bits before making the final decision of the degraded message bits. Then the estimated degraded message bits are once again coded, modulated, and multiplied by the precoder for the degraded part, before being subtracted by the received signal $y_{k}$, i.e., the SIC process. Subsequently, the same iteration process is applied for estimating the designated message bits, as shown in the lower part of Fig. 2.

Denoting the number of bits per symbol, inside each received symbol as $J_{s}$, the extrinsic LLR for the $j_{s}$-th bits $\left(1 \leq j_{s} \leq J_{s}\right.$ ) of the $k$-th user in the $\mu(k)$-th group with received signal $y_{k}$ can be calculated as shown in (28), where $\mathbf{b}_{k}$ is a $J_{s} \times 1$ vector that contains the bits for the $k$-th user, and $s_{\mu(k)}=\operatorname{map}\left(\mathbf{b}_{k}\right)$ denotes the bit-to-symbol mapping function. $\mathbf{b}_{k,\left[j_{s}\right]}$ denotes the sub-vector of $\mathbf{b}_{k}$ without its $j_{s^{-}}$ th bit, and $\mathbf{L}_{A}\left(\mathbf{b}_{k,\left[j_{s}\right]}\right)$ is the $\left(J_{s}-1\right) \times 1$ vector with the $a$ 
priori information of $\mathbf{b}_{k,\left[j_{s}\right]} . \mathbb{B}_{j_{s},+1}$ and $\mathbb{B}_{j_{s},-1}$ represent the set of a total $2^{J_{s}-1}$ bit vectors where its $j_{s}$-th element is either +1 or -1 , respectively. The probability density function for the degraded and designated symbols are in (29) and (30), where $\mathbf{P}_{p}$ denotes the concatenated matrix for all the designated precoders, $\mathbf{s}_{i_{s}}$ denotes the symbol vector which is mapped by a possible combination of designated message bits.

\section{Simulation Results}

This section demonstrates the performance evaluation of the proposed RS precoder in multicarrier environment through comprehensive simulations. First, we compare the achievable fairness rate as a result of using the RS precoder with several selected baseline models including 1 . The representative $3 \mathrm{GPP}$ LTE eMBMS orthogonal transmission scheme [3], equivalent to the SS transmission, without subcarrier allocation (SA), as mentioned in Section II and [18]; 2. No-RS precoding scheme without SA [7], solved via SCA method; 3. NoRS scheme with SA, where $m$ out of $M$ groups are served on each subcarrier, simultaneously; 4. RS-based multicarrier multigroup multicast scheme without SA [29]. Then we investigate the performance improvements achieved by using the RS strategy over the selected benchmark models in terms of coded BER, based on the established transceiver design and linklevel simulation structure. The SNR is equivalent to the total transmit power. We consider a frequency selective independent and identically distributed (i.i.d) Rayleigh fading channels with entries dynamically drawn from $n_{k}^{i} \sim \mathcal{C N}(0,1)$ for each channel realization on each subcarrier, and all the results are obtained by averaging over 1000 realizations. The same 1000 channel realizations that are used in the rate optimization have been imported to the link-level simulator together with the optimized precoder, to guarantee a fair comparison.

\section{A. RS precoder performance under idealistic assumption: Achievable MMF rate}

1) Rate-Splitting vs. Baseline models: Fig.3(a) compares the average achievable MMF group rate, the system configurations are $\left\{K, N_{t}, M, N_{s c}, m\right\}=\{9,4,3,3,2\}$. One can see that, the group MMF rate increases monotonically with SNR, except the baseline mode 2, in which the rate saturation appears (1.8bps/Hz @30dB SNR) due to the lack of the degree of freedom [29]. At the low SNR region, SS without SA (the baseline mode 1) is outperformed by the No-RS scheme (the baseline model 2) with a $2 \mathrm{~dB}$ difference to achieve $1 \mathrm{bps} / \mathrm{Hz}$, as the SS transmission does not benefit from the multiplexing gain. However, when the IGI increases, the SS transmission can outperform the baseline model 2. On the other hand, the gain of using RS over non RS schemes (baseline 1 and 2) is very pronounced, e.g., more than $5 \mathrm{~dB}$ difference to achieve 3bps/Hz group MMF rate. Besides, the proposed RS with SA strikes a good balance between interference management (IGI increases when multiplexing more groups on the same subcarrier) and spectrum efficiency (think of a reduced spectrum efficiency with an orthogonal group-subcarrier mapping), such that it results in a better group MMF rate compare to the baseline mode 4. More obviously at the higher SNR region,

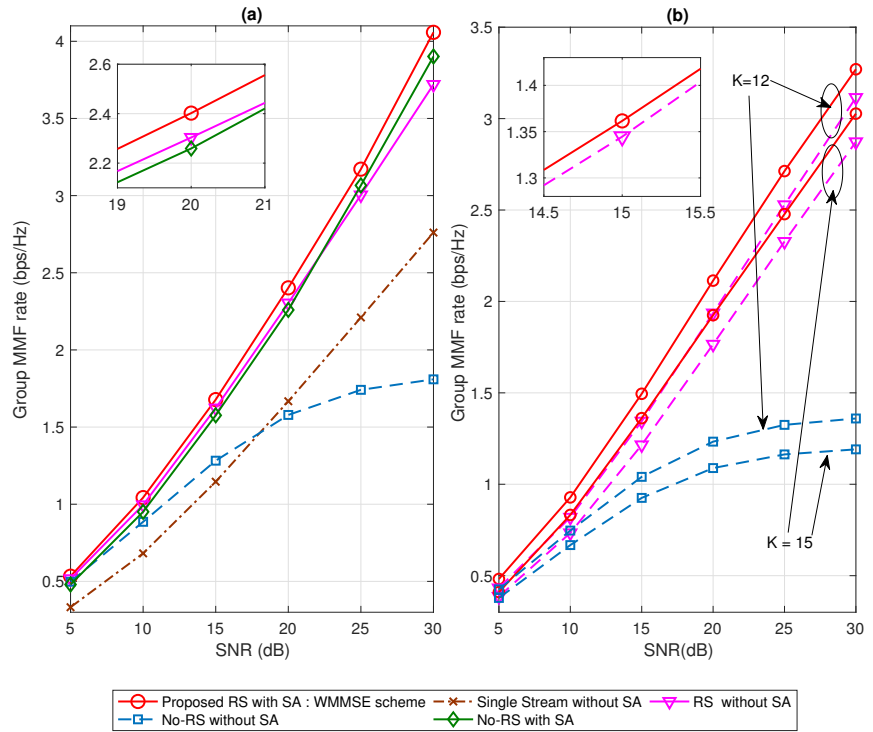

Fig. 3: Group MMF rate vs. SNR for the proposed RS with SA and all four baseline models, (a) $\left\{\mathrm{K}, N_{t}, \mathrm{M}\right\}=\{9,4,3\}$ and (b) $\left\{\mathrm{K}, N_{t}, \mathrm{M}\right\}=\{12 \& 15,4,3\}$

the proposed RS with $\mathrm{SA}$ requires $2 \mathrm{~dB}$ less $\mathrm{SNR}$ to achieve $3.72 \mathrm{bps} / \mathrm{Hz}$, which is the maximum MMF rate that the baseline mode 4 can reach @30dB SNR. With the baseline mode 3 , it can be seen that the SA process helps No-RS avoid rate-saturated by allowing fewer groups multiplexed on each subcarrier. One can also see that the proposed RS with SA slightly outperforms the baseline mode 3 in the whole SNR region tested, with a constant $1 \mathrm{~dB}$ less SNR requirement to achieve the same rate.

2) Increased number of users: In Fig. 3(b), we investigate the average group MMF rate with different system configurations, as we increase the total number of users $K$, e.g., $K=\{12,15\}$. First, as expected, when the total number of users increases, there is a reduction in the achievable group MMF rate of the two proposed methods as well as the baseline models. Secondly, one can observe that with RS (including both with and without SA), the group MMF rate degradation is around $7.5 \%$ when the number of users is increased from 12 to 15 , while without RS, the corresponding loss increases to about $13 \%$. This manifests the fact that with the help of the RS scheme, a fixed amount of the transmission power can be better allocated. It is also evident that, when comparing both configurations, RS with SA can outperform the one without SA by approximately $1.5 \mathrm{~dB}$ SNR to achieve the same MMF rate. We also find that in the SNR region from 5 to $20 \mathrm{~dB}$, the achievable group MMF rate for the case of RS with SA, $\mathrm{K}=15$, is almost the same as the $\mathrm{K}=12$, RS without SA case (difference about $0.02 \mathrm{bps} / \mathrm{Hz}$ at $15 \mathrm{~dB}$ SNR, observed from the zoom-in picture-in-figure). This observation proves that with subcarrier allocation, the frequency diversity is exploited on each subcarrier by discarding the 'struggling' group (i.e., the worst) on that subcarrier, and the absence of that 'bottleneck' group is complemented from other subcarriers. 


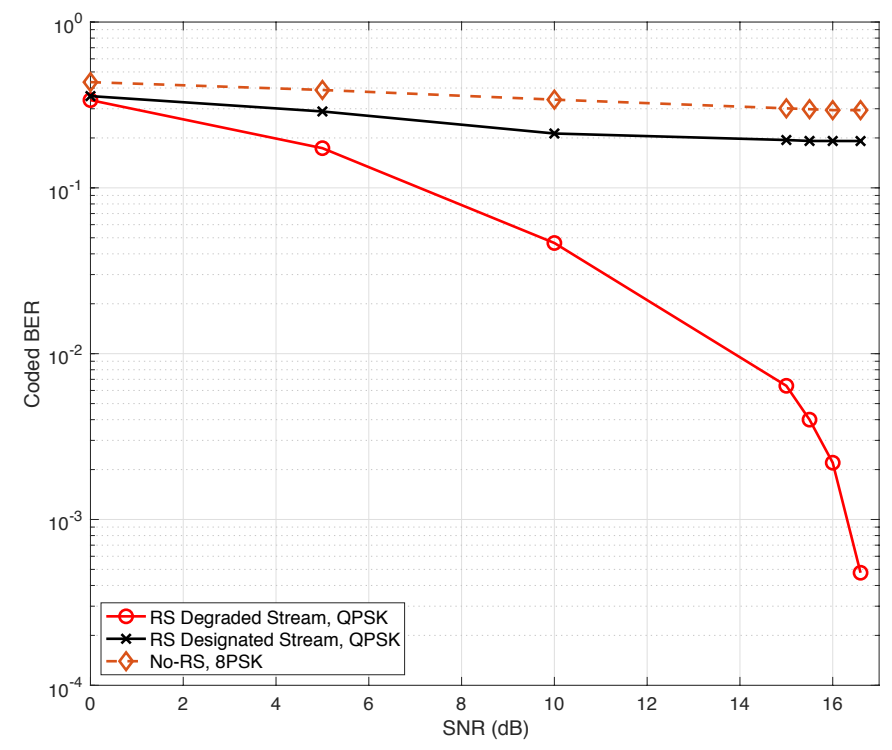

Fig. 4: Coded BER for degraded and designated message of a 2-group multicast system.

\section{B. Coded bit error rate}

In this subsection, the coded BER performance of the RSbased multigroup multicast system is presented. The simulated system is overloaded and has two transmit antennas, and serves two multicast groups simultaneously. Each group has two single-antenna users. The LDPC parity check matrix is generated based on the MATLAB built-in functions for New Radio (NR), the code rate and code length are 0.3235 and 1360, respectively, with an LDPC lifting index of 20. The number of iterations between MAP detector and LDPC decoder is set to 2. To achieve a fair comparison, we consider a traditional No-RS precoding with 8-PSK modulation for each stream, and the WMMSE based RS precoding with QPSK (quadrature phase shift keying) modulation, due tot he fact that RS introduces an extra stream; note that there will always be 6 bits per transmission for the considered case. The lifting index of the LDPC encoder is changed to 24 for the No-RS scheme as it is 8-PSK modulated, but the code rate remains unchanged. The same iterative receiver structure is used for the No-RS scheme, except for the SIC part.

We use BER of $10^{-3}$ as a reference point for the error performance analysis of the considered overloaded multicast system. Results in Fig. 4 shows that the No-RS scheme fails to mitigate the intergroup interference in the overloaded scenario. For the RS scheme, we show two components separately, i.e., degraded and designated streams. The coded BER performance of the No-RS scheme is worse than both components of the RS scheme across the entire tested SNR region. Focusing on the RS scheme, the required SNR for the WMMSE-based RS precoders to reliably receive and decode the degraded part is approximately $16.2 \mathrm{~dB}$. As all the users need to decode the degraded part successfully, the BER of the degraded part is averaged over all groups. However, due to the unbalanced decodability of each group, the average error performance of the designated part is relatively weak, which
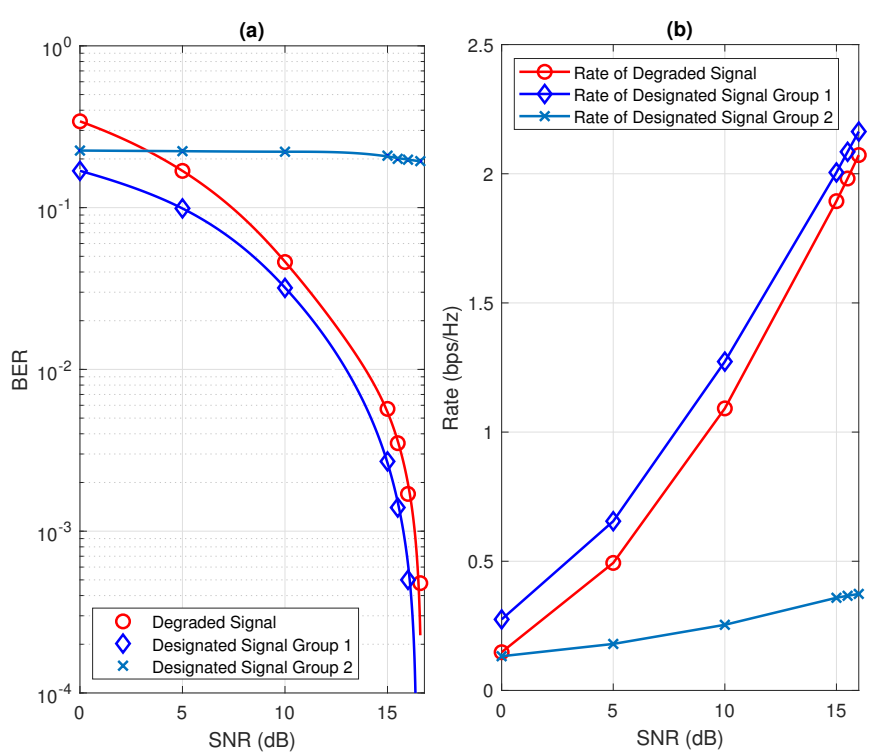

Fig. 5: (a) Coded BER for degraded part and group specific designated part of 2 group multicast with RS, (b) Achievable rate for the degraded part and group specific designated part.

will be further investigated next.

Error performance of individual group: we further look into the error performance of each group separately. As different channel results in different splitting ratio, we stick with one single channel realization. It is observed from Fig. 5(b) that one of the two groups (e.g., Group 1) can achieve a higher designated rate compared to the other group (e.g., Group 2) and it is even higher than the achievable degraded stream rate. Based on this observation, we carry out a group-specific coded BER performance evaluation for the designated part, as shown in Fig. 5(a) together with the coded BER for the degraded part. We can see that for Group 1, it requires approximately $15.8 \mathrm{~dB}$ SNR to reach $10^{-3}$ BER level, which is $0.4 \mathrm{~dB}$ better than the degraded part. This gap on the error performance matches the rate results shown in Fig. 5(b). On the other hand, Group 2 has a high error floor of the designated part and achieves a low rate, correspondingly. The high error floor comes from the Gaussian signalling based rate optimization, where limited power is allocated to the Group 2's designated part. From these results, one can find that, unlike the No-RS scheme where none of the streams can be decoded properly, 2 out of 3 streams in the RS system can be reliably decoded. This exposes the potential of the RS scheme in managing the delivery of different streams, using different coding rates for example, which may further improve its error performance.

\section{CONCLUSIONS}

In this paper, we have investigated the error performance of the rate-splitting based precoding in an overloaded multcarrier multigroup multicast system. We have first proposed a joint RS-based precoding and subcarrier allocation optimization problem, and developed a two-layer WMMSE solution to address the non-convexity of the formulated problem, reaching a optimized RS precoder design by using alternative optimization method. Then we have evaluated the error performance 
of the RS system through an established transceiver structure, taking into account the optimal RS precoder from the rate optimization. From the coded BER results, it has been demonstrated that the benchmark schemes such as the traditional linear precoding cannot properly decode any of the streams and results in a high error probability. In contrast, with RS, the degraded stream as well as a certain amount of the designated streams, can be decoded reliably. We have also provided the insight that the decoding ability and error performance of the RS scheme match its corresponding rate results.

Our finding reveals the potential of deploying RS in a practical systems, leveraging on the transceiver structure and evaluation framework that established in this paper.

\section{REFERENCES}

[1] H. Chen, D. Mi, Z. Chu, P. Xiao, Y. Xu, and D. He, "Link-Level Performance of Rate-Splitting based Downlink Multiuser MISO Systems," in IEEE 31st Annual International Symposium on Personal, Indoor and Mobile Radio Communications (PIMRC), Aug. 2020, pp. 1-5.

[2] M. Fuentes, D. Mi, H. Chen, E. Garro, J. L. Carcel, D. Vargas, B. Mouhouche, and D. Barquero, "Physical Layer Performance Evaluation of LTE-Advanced Pro Broadcast and ATSC 3.0 Systems," IEEE Transactions on Broadcasting, vol. 65, no. 3, pp. 477-488, Sep. 2019.

[3] D. Vargas, D. Mi (Eds), "LTE-A Pro Broadcast Radio Access Network Benchmark," 5G-PPP 5G-Xcast Project, Deliverable D3.1, Nov. 2017.

[4] 3GPP, "Evolved Universal Terrestrial Radio Access (E-UTRA); Study on LTE-based 5G terrestrial broadcast (Release 16)," 3rd Generation Partnership Project (3GPP), Technical Report (TR) 36.776, Mar. 2019.

[5] RP-193248, "New Work Item on NR support of Multicast and Broadcast Services," Huawei, 3GPP TSG RAN \#86, Dec. 2019.

[6] M.J.Lopez, "Multiplexing, scheduling, and multicasting strategies for antenna arrays in wireless networks," Ph.D. dissertation, Dept. Elect. Eng. and Comp. Sci., MIT,Cambridge, 2002.

[7] N. D. Sidiropoulos, T. N. Davidson, and Z.-Q. Luo, "Transmit beamforming for physical-layer multicasting," IEEE Transactions on Signal Processing, vol. 54, no. 6, pp. 2239-2251, Jun. 2006.

[8] B. Gopalakrishnan and N. D. Sidiropoulos, "High performance adaptive algorithms for single-group multicast beamforming," IEEE Transactions on Signal Processing, vol. 63, no. 16, pp. 4373-4384, Aug 2015.

[9] I. H. Kim, D. J. Love, and S. Y. Park, "Optimal and successive approaches to signal design for multiple antenna physical layer multicasting," IEEE Transactions on Communications, vol. 59, no. 8, pp. 2316-2327, Aug. 2011.

[10] R. Hunger, D. A. Schmidt, M. Joham, A. Schwing, and W. Utschick, "Design of single-group multicasting-beamformers," in IEEE International Conference on Communications, Jun. 2007, pp. 2499-2505.

[11] B. Du, Y. Jiang, X. Xu, and X. Dai, "Transmit beamforming for mimo multicast channels," in IEEE International Conference on Communications, Jun. 2012, pp. 3800-3805.

[12] E. Karipidis, N. D. Sidiropoulos, and Z.-Q. Luo, "Transmit beamforming to multiple co-channel multicast groups," in IEEE 1st International Workshop on CAMAP, 2005., Dec. 2005, pp. 109-112.

[13] _ - "Quality of service and max-min fair transmit beamforming to multiple cochannel multicast groups," IEEE Transactions on Signal Processing, vol. 56, no. 3, pp. 1268-1279, Mar. 2008.

[14] — , "Far-field multicast beamforming for uniform linear antenna arrays," IEEE Transactions on Signal Processing, vol. 55, no. 10, pp. 4916-4927, Oct. 2007.

[15] D. Christopoulos, S. Chatzinotas, and B. Ottersten, "Weighted fair multicast multigroup beamforming under per-antenna power constraints," IEEE Transactions on Signal Processing, vol. 62, no. 19, pp. 51325142, Aug. 2014.

[16] N. D. Sidiropoulos and T. N. Davidson, "Broadcasting with channel state information," in IEEE Sensor Array and Multichannel Signal Processing Workshop, Jul. 2004, pp. 489-493.

[17] J. Li and A. P. Petropulu, "On transmit beamforming for physical-layer multicasting," in IEEE Global Telecommunications Conference, Dec. 2011, pp. 1-5.

[18] H. Joudeh and B. Clerckx, "A rate-splitting strategy for max-min fair multigroup multicasting," in IEEE 17th International Workshop on SPAWC, Jul. 2016, pp. 1-5.
[19] _ - "Rate-splitting for max-min fair multigroup multicast beamforming in overloaded systems," IEEE Transactions on Wireless Communications, vol. 16, no. 11, pp. 7276-7289, Nov. 2017.

[20] D. Christopoulos, S. Chatzinotas, and B. Ottersten, "Multicast multigroup precoding and user scheduling for frame-based satellite communications," IEEE Transactions on Wireless Communications, vol. 14, no. 9 , pp. 4695-4707, Sep. 2015

[21] Y. Mao, B. Clerckx, and V. O. K. Li, "Energy Efficiency of RateSplitting Multiple Access, and Performance Benefits over SDMA and NOMA," in 15th International Symposium on Wireless Communication Systems (ISWCS), Aug. 2018, pp. 1-5.

[22] Y. Mao, B. Clerckx, and V. O. K. Li, "Rate-splitting multiple access for downlink communication systems: Bridging, generalizing, and outperforming SDMA and NOMA," EURASIP J. Wireless Commun. Netw., vol. 2018, no. 1, p. 133, May 2018.

[23] H. Joudeh and B. Clerckx, "Sum-rate maximization for linearly precoded downlink multiuser MISO systems with partial CSIT: A rate-splitting approach," IEEE Transactions on Communications, vol. 64, no. 11, pp. 4847-4861, Nov. 2016.

[24] Y. Mao, B. Clerckx, and V. O. K. Li, "Rate-splitting for multi-antenna non-orthogonal unicast and multicast transmission: Spectral and energy efficiency analysis," IEEE Transactions on Communications, vol. 67, no. 12 , pp. 8754-8770, 2019.

[25] H. Joudeh and B. Clerckx, "Robust Transmission in Downlink Multiuser MISO Systems: A Rate-Splitting Approach," IEEE Transactions on Signal Processing, vol. 64, no. 23, pp. 6227-6242, Dec. 2016.

[26] Y. Mao, B. Clerckx, J. Zhang, V. O. K. Li, and M. A. Arafah, "Max-Min Fairness of K-User Cooperative Rate-Splitting in MISO Broadcast Channel With User Relaying," IEEE Transactions on Wireless Communications, vol. 19, no. 10, pp. 6362-6376, Oct. 2020.

[27] W. Dang, M. Tao, H. Mu, and J. Huang, "Subcarrier-pair based resource allocation for cooperative multi-relay ofdm systems," IEEE Transactions on Wireless Communications, vol. 9, no. 5, pp. 1640-1649, May 2010.

[28] D. W. K. Ng, E. S. Lo, and R. Schober, "Energy-efficient resource allocation in multi-cell ofdma systems with limited backhaul capacity," IEEE Transactions on Wireless Communications, vol. 11, no. 10, pp. 3618-3631, Oct. 2012.

[29] H. Chen, D. Mi, Z. Chu, P. Xiao, and R. Tafazolli, "Rate-splitting for multigroup multicast beamforming in multicarrier systems," in 2018 IEEE 19th International Workshop on SPAWC, Jun. 2018, pp. 1-5.

[30] H. Chen, D. Mi, B. Clerckx, Z. Chu, J. Shi, and P. Xiao, "Joint Power and Subcarrier Allocation Optimization for Multigroup Multicast Systems with Rate Splitting," IEEE Transactions on Vehicular Technology, pp. $1-1,2019$.

[31] N. D. Sidiropoulos, T. N. Davidson, and Z.-Q. Luo, "Transmit beamforming for physical-layer multicasting," IEEE Transactions on Signal Processing, vol. 54, no. 6, pp. 2239-2251, Jun. 2006.

[32] A. Schad and M. Pesavento, "Max-min fair transmit beamforming for multi-group multicasting," in International ITG Workshop on Smart Antennas (WSA), Mar. 2012, pp. 115-118.

[33] O. Dizdar, Y. Mao, W. Han, and B. Clerckx, "Rate-splitting multiple access for downlink multi-antenna communications: Physical layer design and link-level simulations," in IEEE 31st PIMRC, Aug. 2020, pp. 1-6.

[34] Y. Wu, M. Wang, C. Xiao, Z. Ding, and X. Gao, "Linear precoding for MIMO broadcast channels with finite-alphabet constraints," IEEE Transactions on Wireless Communications, vol. 11, no. 8, pp. 29062920, Aug. 2012.

[35] Y. Wu, C. Xiao, X. Gao, J. D. Matyjas, and Z. Ding, "Linear precoder design for MIMO interference channels with finite-alphabet signaling," IEEE Transactions on Communications, vol. 61, no. 9, pp. 3766-3780, Sep. 2013.

[36] B. M. Hochwald and S. ten Brink, "Achieving near-capacity on a multiple-antenna channel," IEEE Transactions on Communications, vol. 51, no. 3, pp. 389-399, Mar. 2003. 